\title{
MICROBIAL COMMUNITY STRUCTURE AND SEASONAL VARIATIONS IN MUDFLAT SEDIMENTS OF SANSHA BAY, CHINA
}

\author{
YANG, Z. ${ }^{1}-$ WANG, Y. Z. ${ }^{1}-$ WU, Z. C. ${ }^{1}-$ REN, L. R. ${ }^{1}-$ XIONG, C. J. ${ }^{2}-$ MA, Y.${ }^{1 *}$ \\ ${ }^{1}$ Key Laboratory of Healthy Mariculture for the East China Sea, Ministry of Agriculture, \\ Fisheries College of Jimei University, Xiamen 361021, China \\ ${ }^{2}$ College of Foreign Language, Hunan University, Changsha 410006, China \\ *Corresponding author \\ e-mail: maying@jmu.edu.cn; phone: +86-1896-5423-006; fax: +86-0592-6181-476
}

(Received $13^{\text {th }}$ Jul 2019; accepted $25^{\text {th }}$ Nov 2019)

\begin{abstract}
Illumina high-throughput sequencing was applied to study the microbial community structure and seasonal variations in the mudflat sediments of Sansha Bay, China. Significant seasonal differences of microbial communities were observed, and $\mathrm{pH}$, total $\mathrm{P}$, total $\mathrm{N}$, and organic carbon concentration were important factors affecting the microbial communities. A total of 52 bacterial phyla were detected in the sediments, dominated by $\gamma$-Proteobacteria $(27.78 \%)$, $\delta$-Proteobacteria $(20.16 \%)$ and Bacteroidetes $(10.58 \%)$. A total of 717 bacterial genera were detected, and among the top 100 genera with the highest abundances, 48 genera changed significantly $(p<0.05)$ with seasons. The microbial community structure in summer was significantly different from others, and the environmental factors driving the changes were mainly temperature, organic carbon and total $\mathrm{N}$ concentration. The bacteria with higher abundances in summer were mainly attached bacteria, thermophilic bacteria, anaerobic bacteria, and those involved in carbon and nitrogen cycling, while in other seasons, those with higher abundances were mainly aerobic bacteria, which could adapt to low temperatures and are involved in the degradation of cellulose and chitosan. However, the physicochemical indexes didn't change significantly, which indicated that the bacterial communities are more sensitive and can serve as a good sentinel for environmental changes.

Keywords: Illumina high-throughput sequencing, bacterial genetic diversity, seasonal changes, intertidal sediments, physiochemical characteristics, Sansha Bay
\end{abstract}

\section{Introduction}

Traditionally, marine environmental quality is assessed by physical-chemical and biological indexes, such as dissolved oxygen (DO), chemical oxygen demand (COD), nitrogen $(\mathrm{N})$, phosphorus (P), heavy metal, persistent pollutant, and so on (Arsad et al., 2012). However, such static indexes have difficulties characterizing the structure and function of the marine ecosystem (Viswanathan et al., 2010). At present, most of the biological indicators used to characterize ecosystem structure and function are large animals, plants or benthos (Cabana et al., 2013; Hannah, 2015; Wang et al., 2018), or some microscopically visible single-celled organisms, such as diatoms and cyanobacteria (Daby, 2006). Nevertheless, when the impact of the disturbance has caused obvious changes in these biological indicators, the time of early warning is long gone.

Microorganisms are one of the most important components of the intertidal zones of mudflat ecosystems, and are involved in nutrient cycling, energy flow, organic matter degradation, and pollutant removal. Microbial communities are sensitive to environmental variation, which makes them ideal biological indicators for environmental pollution and ecosystem changes (Sun et al., 2012). Total coliforms, 
fecal coliforms (Escherichia coli) and enterococci have been commonly used as indicators to assess the microbiological safety of water resources (Lage and Bondoso, 2011). Previous studies also showed that there was a positive and strong association of total phosphorous, total nitrogen, and ammonium-nitrogen contents with microeukaryotes and Gram-positive bacteria (Zhao et al., 2010), and several metals are also strongly associated with microbial community composition (Cao et al., 2006). Although microorganisms are more sensitive to environmental changes and are better indicators for environmental quality, they have not been adopted as environmental quality indexes yet due to our poor understanding of them and the difficulty of detection. The emergence of molecular biological technique, especially the highthroughput sequencing, has provided us important opportunity for fully and deeply understand environmental microbes because of the high data throughput $\left(10^{3}-10^{6}\right.$ sequences per sample), high accuracy and low cost. This technique enables us to identify the dominant and rare populations within a community simultaneously, thus it could reveal a significantly greater level of microbial diversity than conventional molecular tools (Binladen et al., 2007; Caporaso and Gordon, 2011).

Sansha Bay is a typical enclosed bay, which is located on the southeastern coast of Ningde City, Fujian Province, and includes Dongwuyang, Guanjingyang, and Sanduao. The streams and rivers from the eight surrounding cities, with a basin area of $8,700 \mathrm{~km}^{2}$, flow into this harbor, containing the domestic sewage of over 2 million people (Tang et al., 2018). In recent years, due to the high discharge of domestic sewage and industrial wastewater as well as the large inflow of residual breeding bait, the mudflat environment has been subjected to substantial pollution. The main goals of the study are to investigate the community structure and seasonal variations of organisms in mudflat sediments of Sansha Bay, and their relationship to physicochemical indexes. The results present here will provide microbial parameters for assessing the environment quality, and to lay a foundation for further illumination of the ecological functions of microorganisms in mudflat sediments.

\section{Methods}

\section{Sample collection and physiochemical index analysis}

The study was performed in the Northern Yantian port of Sansha Bay (Fig. Al). The total area was about 2.6 acre, at an east longitude from 119.7975 to 119.7986 and a northern latitude from 26.8411 to 30.8422 . The study area was divided into four plots, and sediment samples of each plot were collected on 10 October, 2014 (autumn), 2 January, 2015 (winter), 6 April 2015 (spring), and 2 July 2015 (summer). The sediments of the surface layer (top 3-5 cm) were removed and about $200 \mathrm{~g}$ subsurface sediments from three different sites within the same plot were collected and mixed as one sample, resulting in a total of 16 samples in four batches. The samples were termed as Oct-P1 to Oct-P4, Jan-P1 to Jan-P4, Apr-P1 to Apr-P4, Jul-P1 to Jul-P4, according to sampling time and sampling plots.

All the 16 sediment samples were used for the determination of the physicochemical indices, $10 \mathrm{~g}$ sediments of each sample were freeze-dried in a lyophilizer (Labconco, Kansas City, MO, USA). The following parameters were analyzed, and each sample was measured twice. Total nitrogen (TN) was determined via the Kjeldahl method (Bradstreet, 1954) and total phosphorus (TP) via the ammonium molybdate spectrophotometric method (GB11894-89, China; Huang, 
2000). Organic matter contents (OrgC and OrgS) were determined via a Vario Max CNS analyzer (Elementar, Hanau, Germany). For the determination of $\mathrm{pH}$, sediment and water were mixed at a ratio of 1:5 (W/V). Water temperature $(\mathrm{Tm})$ was measured on the site.

\section{DNA extraction, PCR amplification, and Illumina sequencing}

For DNA extraction with the PowerSoil DNA Isolation Kit (MOBIO-Laboratories, USA), we used $0.25 \mathrm{~g}$ of the sediment samples. The V4 variable region in the bacterial $16 \mathrm{~S}$ rRNA gene was amplified with the primer pair 515F (5'- GTG CCA GCM GCC GCG GTA A-3') and 806R (5'- GGA CTA CHV GGG TWT CTA AT-3'). The PCR products of the different samples were mixed with equimolar concentration and sent to the Majorbio Co. Ltd. (Shanghai) for paired-end sequencing through the Illumina Miseq platform.

\section{Bioinformatic analysis}

Post-processing of the Illumina sequence reads included quality control and clustering of the operational taxonomic unit (OTU) with $97 \%$ sequence identity. The QIIME was applied to collect the OTUs for sequence clustering, the chimeras were removed, and OTU abundance was defined. The RDP Bayesian classifier was applied to conduct taxonomic analyses on OUT-representative sequences with $97 \%$ similarity level; the classification was identified in the Silva database (Release128 http://www.arb-silva.de), and the community composition of each sample was analyzed at each classification level, including phylum, class, order, family, genus, and OTU.

Mothur software was used to analyze alpha diversity. Community diversity was estimated by Shannon (Magurran, 1988) and Simpson (Simpson, 1949) indices. The total number of species in each sample was estimated with Chao1 and Ace. Phylogenetic diversity (PD) whole tree was used to analyze the relationships among observed species. Sampling coverage was used to evaluate whether the sequencing quantity is enough.

Hierarchical clustering was analyzed by the unweighted pair group method with arithmetic (UPGMA). Student's $t$ - test was used to test the significant difference of alpha diversity in different season, and one-way ANOVA was used to test the differences of physicochemical factors or bacterial communities at the phylum and genus levels. All statistical analyses were carried out using software SPSS 18.0 or stats packages in the $\mathrm{R}$ environment (version 3.2.2, http://www.r-project.org) ( $\mathrm{R}$ Development Core Team, 2008).

Redundancy Analysis (RDA) was used to reveal the relationship between physicochemical factors and bacterial community composition (Wollenberg, 1977). Forward selection of physicochemical factors was performed by Monte Carlo test with 999 permutations. The Partial Mantel test was also applied to distinguish the relative contribution of time variables (Legendre et al., 2016). RDA was carried out using rda packages in $\mathrm{R}$ environment. The original sequence data obtained was submitted to the NCBI SRA database with the registration number PRJNA505598. 


\section{Results}

\section{Sequencing results and alpha diversity analysis}

We obtained a total of 533,568 effective high-quality sequences, which were classified into 65,517 OTUs, with an average of 4,095 \pm 346.52 OTUs in each sample. Sequence homogenization was conducted to obtain 30,000 sequences in each sample, followed by alpha diversity analysis; the results are shown in Table 1. The coverage of each sample was over $96 \%$, indicating that most bacterial groups could be determined. However, the sequencing depth did not reach saturation, which indicates that the microflorae in the sediment of Sansha Bay was highly abundant. The Shannon index values ranged from 6.31 to 7.02, while the PD index varied between 179.61 and 242.73. The diversity index variations in each sample were not significantly different $(p>0.05$, Table Al) among the different seasons.

Table 1. Alpha diversity indicators (OTU, Shannon, Simpson, ACE, Chaol, PD and coverage) of bacterial communities in mudflat sediments of Sansha Bay

\begin{tabular}{c|c|c|c|c|c|c|c|c}
\hline $\begin{array}{c}\text { Sample } \\
\text { name }\end{array}$ & OTUs & Shannon & Simpson & ACE & Chao1 & PD & Coverage & $\begin{array}{c}\text { Sampling } \\
\text { date }\end{array}$ \\
\hline Oct-P1 & 4056 & 6.70 & 0.0050 & 5717.53 & 5640.81 & 218.68 & 0.96 & \\
Oct-P2 & 4388 & 6.85 & 0.0042 & 6145.69 & 6067.05 & 232.08 & 0.96 & October 10, 2014 \\
Oct-P3 & 3877 & 6.57 & 0.0057 & 5537.69 & 4987.95 & 207.8 & 0.96 & \\
Oct-P4 & 3662 & 6.61 & 0.0048 & 5073.19 & 5384.34 & 195.33 & 0.97 & \\
Average & 3996 & 6.68 & 0.0049 & 5618.53 & 5520.04 & 213.47 & 0.96 & \\
\hline Jan-P1 & 4366 & 6.84 & 0.0041 & 6065.44 & 6336.64 & 229.15 & 0.96 & \\
Jan-P2 & 4074 & 6.61 & 0.0065 & 5654.31 & 6266.61 & 215.66 & 0.96 & January 2, 2015 \\
Jan-P3 & 4093 & 6.65 & 0.0055 & 5719.33 & 5590.49 & 214.78 & 0.96 & \\
Jan-P4 & 4695 & 7.02 & 0.0036 & 6220.91 & 5766.58 & 242.73 & 0.96 & \\
Average & 4307 & 6.78 & 0.0049 & 5915.00 & 5990.08 & 225.58 & 0.96 & \\
\hline Apr-P1 & 3615 & 6.37 & 0.0073 & 5344.25 & 5611.13 & 187.63 & 0.96 & \\
Apr-P2 & 4199 & 6.73 & 0.0053 & 5628.99 & 5205.31 & 218.65 & 0.96 & \\
Apr-P3 & 4349 & 6.70 & 0.0060 & 6031.25 & 5981.72 & 226.72 & 0.96 & April 6, 2015 \\
Apr-P4 & 3875 & 6.63 & 0.0052 & 5620.25 & 5765.90 & 207.17 & 0.96 & \\
Average & 4010 & 6.61 & 0.0060 & 5656.19 & 5641.01 & 210.04 & 0.96 & \\
\hline Jul-P1 & 4332 & 6.83 & 0.0044 & 5990.67 & 6200.28 & 226.44 & 0.96 & \\
Jul-P2 & 4363 & 6.92 & 0.0038 & 5853.22 & 6056.11 & 223.84 & 0.96 & \\
Jul-P3 & 4218 & 6.87 & 0.0037 & 5990.22 & 6040.11 & 220.84 & 0.96 & July 2, 2015 \\
Jul-P4 & 3355 & 6.31 & 0.0077 & 4816.68 & 4801.49 & 179.61 & 0.97 & \\
Average & 4067 & 6.73 & 0.0049 & 5662.70 & 5774.49 & 212.68 & 0.96 & \\
\hline
\end{tabular}

Oct, Jan, Apr and Jul indicate sampling times; P1, P2, P3 and P4 represent four different sampling plots. OUT represent operational taxonomic unit, PD represent phylogenetic diversity

\section{Beta diversity}

According to the beta diversity distance matrix, hierarchical clustering analysis was conducted at different classification levels, and the UPGMA algorithm was used to construct the tree structure (Fig. 1). Figure 1 shows that the clustering results were not stable at the phylum and class levels. However, from the order to the OTU level, except for sample Jan-P4, the other samples were clustered into four categories according to the sampling time; the most significant difference was observed between the summer sample and other samples, while the differences were lower among spring, autumn, and winter samples. 

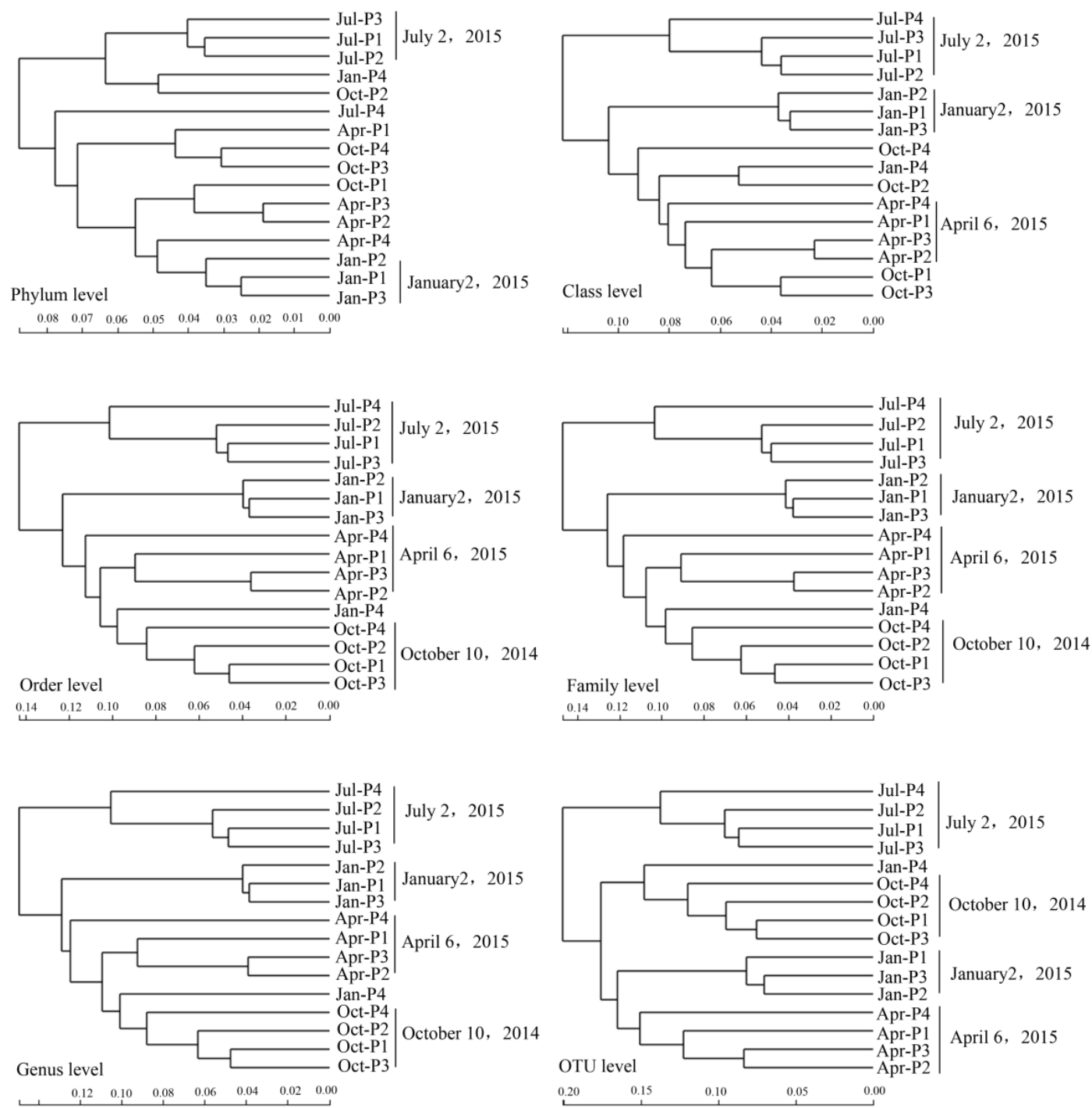

Figure 1. Clustering analysis diagram of mudflat sediment samples from Sansha Bay, China (Oct, Jan, Apr and Jul indicate sampling times; P1, P2, P3 and P4 represent four different sampling plots)

\section{Microbial community structure and seasonal changes in mudflat sediments in Sansha Bay}

Microbial community structure and dynamic changes at the phylum level

We detected a total of 52 bacterial phyla in the mudflat sediments of Sansha Bay (Fig. 2). The dominant microbial phylum was Proteobacteria, and the proportions of $\gamma$-Proteobacteria, $\delta$-Proteobacteria and $\alpha$-Proteobacteria in the total sequences were 27.78, 20.16 and 4.36\%, respectively. The subdominant phylum Bacteroidetes accounted for $10.58 \%$ of the total sequences. The other abundant phyla were Planctomycetes (5.09\%), Chloroflexi (4.95\%), Acidobacteria (3.65\%), Nitrospirae (2.38\%), Cyanobacteria (2.10\%), Gemmatimonadetes (1.94\%), Verrucomicrobia $(1.59 \%)$, and Latescibacteria $(1.01 \%)$. The proportions of the other 42 bacterial phyla, including Spirochaetae, Actinobacteria, and Ignavibacteriae, were always below $1 \%$. 


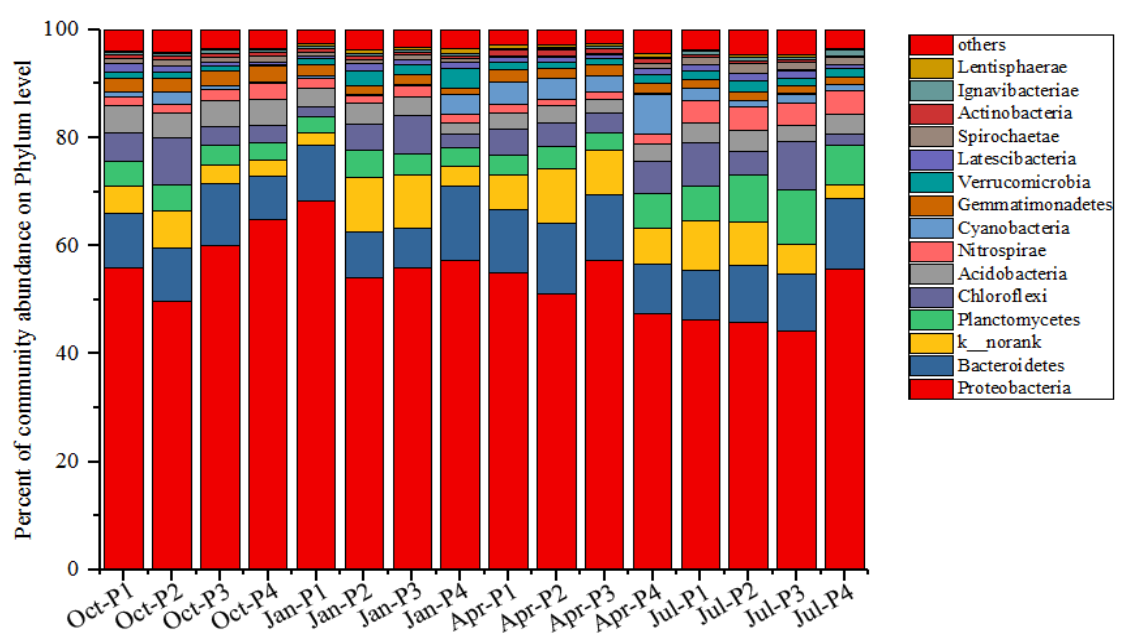

Figure 2. Microbial communities in mudflat sediments of Sansha Bay, China, at the phylum level (Oct, Jan, Apr and Jul indicate sampling times; P1, P2, P3 and P4 represent four different sampling plots)

Of the 52 bacterial phyla, 14 showed significant differences $(p<0.05$, Table A2) among the different sampling seasons. Compared with the other seasons, the abundances of nine phyla were significantly increased in summer, including Planctomycetes, Nitrospirae, Spirochaetae, Ignavibacteriae, Hydrogenedentes, KSB3_Modulibacteria, Chlorobi, Tenericutes, and PAUC34f. In contrast, the abundances of two phyla, Actinobacteria and Armatimonadetes, were significantly increased in winter.

Microbial community structure and dynamic changes at the genus level

A total of 717 bacterial genera were detected in the sediments of Sansha Bay. The dominant microbial genus that could be identified was Desulfobulbus, which occupied 4.97\%. The subdominant genus were Nitrospira (2.34\%) and Sva0081_sediment_group (1.73\%). Most of the genera occupied less than 1\%, including Halioglobus, Draconibacterium, Deferrisoma, Spirochaeta, Urania-1B-19_marine_sediment_group, Haliea, Desulfatiglans, Aquibacter and so on. In addition, unclassified genera also occupied a large proportion, such as c_Gammaproteobacteria (occupied 6.69\%), f_JTB255_marine_benthic_group $(6.22 \%)$ and o_Gammaproteobacteria Incertae_Sedis $(4.32 \%)$, etc.

Among the top 100 genera with the highest abundances, 48 genera showed significant variations ( $p<0.05$, Table A3). The clustering results of the heat map (Fig. 3) also indicated significant seasonal differences in the microbial community structures, with more significant differences between summer and other seasons and smaller differences among spring, autumn, and winter. Compared with the samples from the other seasons, in summer, 13 genera showed significantly higher abundances, including Nitrospira, f_Flammeovirgaceae, Deferrisoma, Spirochaeta and f_Lentimicrobiaceae, etc., while in winter, genera Aquibacter, Kangiella, Lutibacter and other 9 genera increased significantly. In autumn, 14 genera increased significantly, including Haliea, Desulfatiglans, Sulfurovum, Robiginitalea, and Haliangium, etc., while only Halioglobus and other 8 genera increased significantly in spring. 


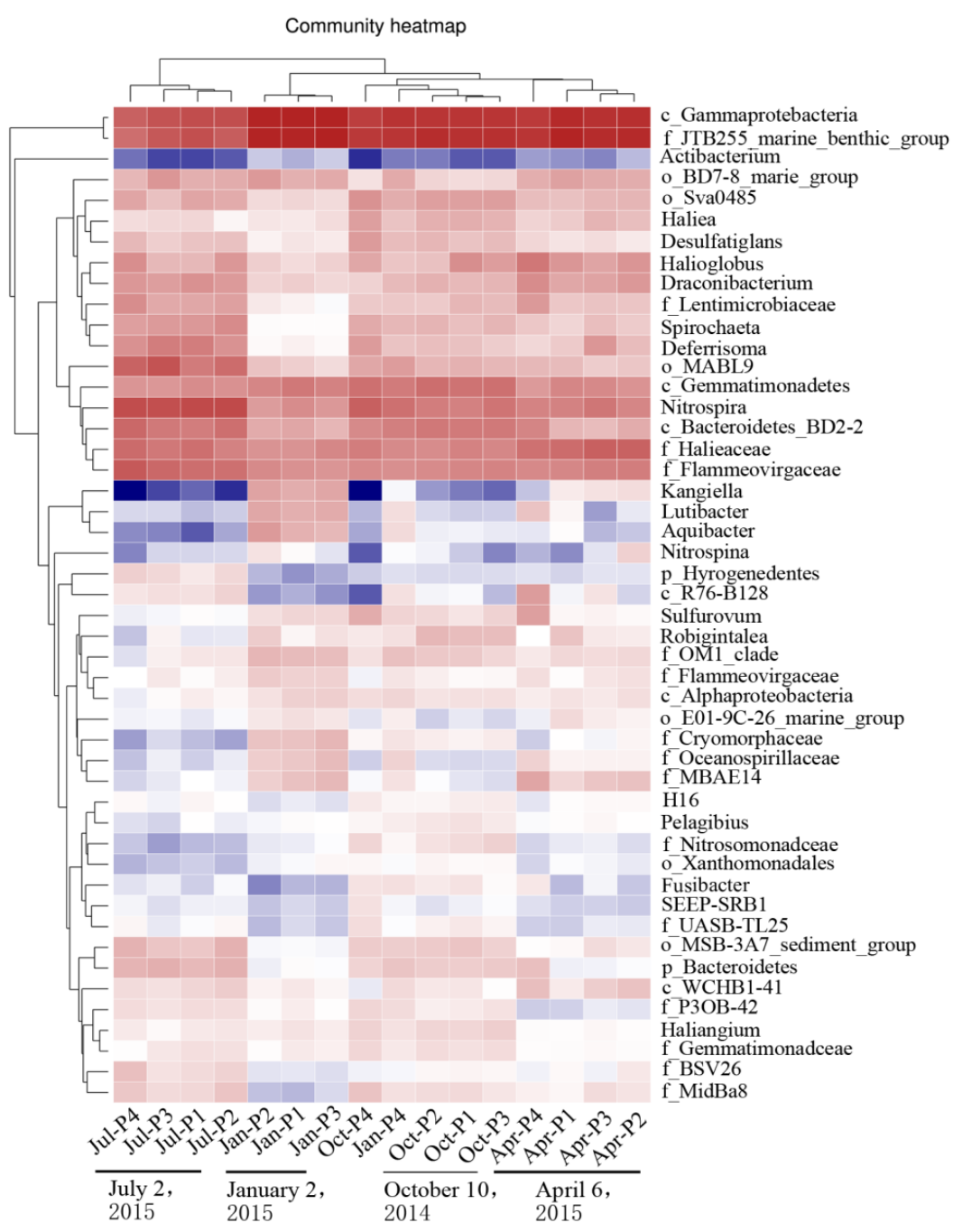

Figure 3. Heatmap of the microbial communities in mudflat sediments of Sansha Bay, China, at the genus level. (Oct, Jan, Apr and Jul indicate sampling times; P1, P2, P3 and P4 represent four different sampling plots)

Physicochemical indices of mudflat sediments in Sansha Bay and the relationships with microbial communities

The values of the physicochemical indices are shown in Figure 4. In the different sampling seasons, $\mathrm{pH}$ ranged from 8.37 to 8.59 , with highest values in spring and lowest values in winter. Temperature varied between 12.61 and $30.06^{\circ} \mathrm{C}$, with highest values in summer and lowest values in winter, and the difference between summer and winter had reached a significant level ( $p<0.05$, Table A4). Furthermore, organic carbon, total nitrogen, and total phosphorus concentrations were also highest in summer, while the organic sulfur levels were lowest in summer and highest in winter. However, except for the temperature, all these differences among other physicochemical indices were not statistically significant.

At the genus level, the microbial communities were used as indicators for environmental changes applying redundancy analysis (RDA), and six physicochemical indices of the sediment, including $\mathrm{TP}, \mathrm{TN}, \operatorname{OrgC}, \operatorname{OrgS}, \mathrm{Tm}$, and $\mathrm{pH}$, were used as 
explanatory variables (Fig. 5). The RDA1 and RDA2 explained 76.48 and $3.74 \%$ of the total variance, respectively. At the genus level, the microbial community structure in summer differed most significantly from those of the other seasons, followed by the community structure in spring. In contrast, the communities in winter and autumn showed a higher similarity. TP, TN, OrgC, Tm, and $\mathrm{pH}$ were important factors affecting the microbial community structure and were correlated $(p<0.05)$ or highly correlated $(p$ $<0.01)$ with microbial community structure (Table A5).
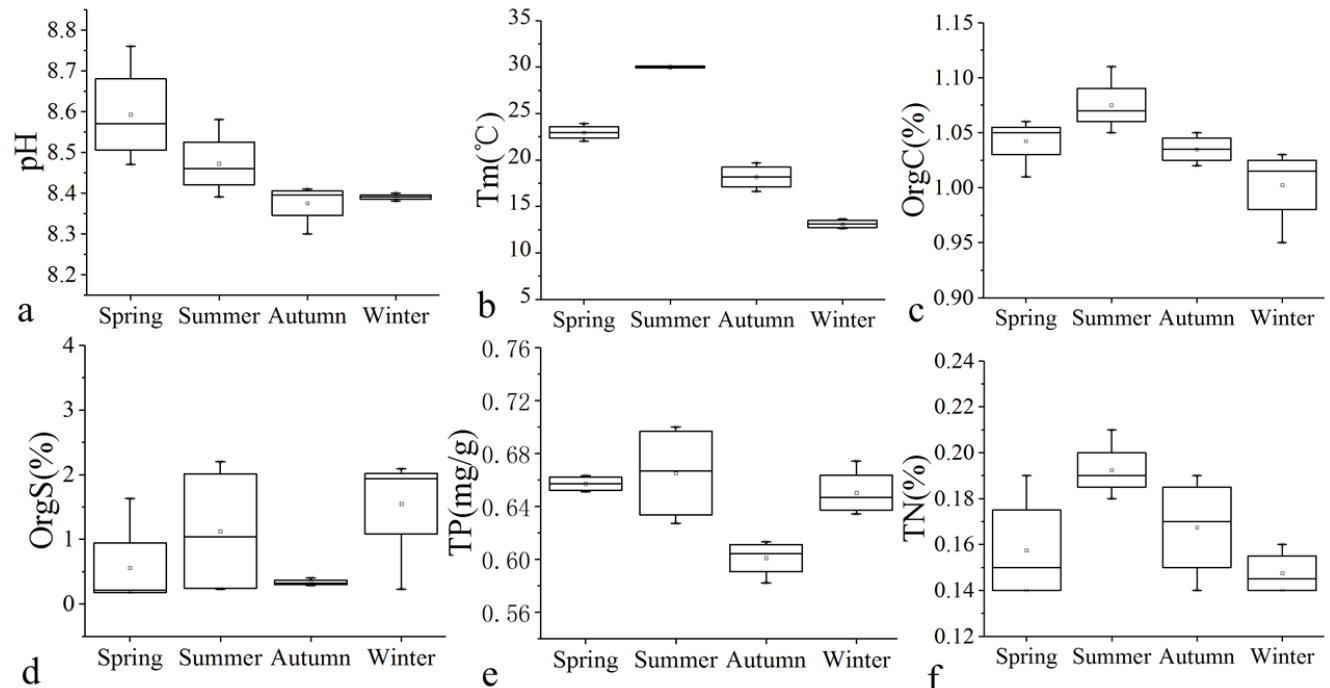

Figure 4. Physicochemical indices of mudflat sediments in Sansha Bay, China. (a) pH, (b) Temperture $\left({ }^{\circ} \mathrm{C}\right)$, (c) Organic carbon (\%), (d) Organic sulfur (\%), (e) Total phosphorus $(\mathrm{mg} / \mathrm{g})$, (f) Total nitrogen (\%)

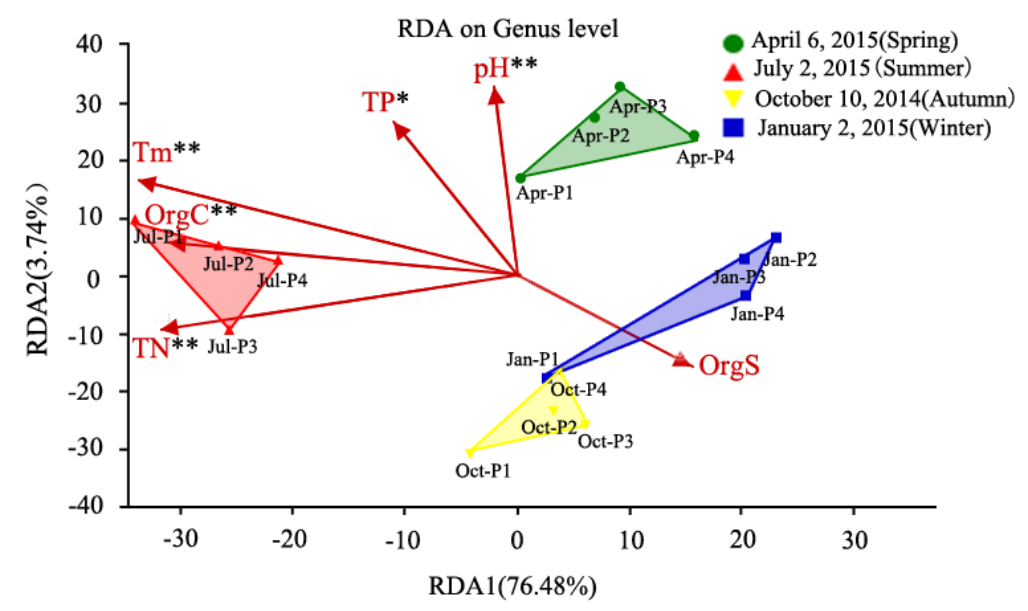

Figure 5. Relationships between microbial communities and environmental factors in Sansha Bay sediments

(TN, TP, OrgC, OrgS and Tm represent total nitrogen, total phosphorus, Organic carbon, sulfur contents and water temperature, respectively. Oct, Jan, Apr and Jul indicate sampling times;

$P 1, P 2, P 3$ and $P 4$ represent four different sampling plots. Arrow direction represents the direction of increasing environmental factors. * and ** represent significance levels of 0.05 and 0.01 , respectively) 


\section{Discussion}

Previous studies have indicated temperature is an important factor influencing microbial diversity in mudflat sediments (Hicks et al., 2018). The seasonal differences of microorganisms in mudflat sediments of Sansha Bay were significant, and the summer samples most significantly differed from the samples taken in the other seasons. This was consistent with the significantly higher water temperature in Sansha Bay at summer, indicating a highly significant correlation between temperature and microbial communities. Temperature was also an important factor affecting phytoplankton communities, and in a previous study, the phytoplankton density increased with increasing water temperatures (Wang et al., 2015). While, the higher phytoplankton density results in the release of higher amounts of dissolved organic matter, directly affecting the microbial community structure (Shabarova et al., 2017). Also it can be seen in Fig. 5, TN and OrgC were significantly correlated with the microbial community structure and had the highest impacts on microbial communities in summer.

In terms of microbial community composition, the abundance of Planctomycetes was significantly higher in summer, and most of them are attached bacteria (Caporaso and Gordon, 2011). The significant increase of such bacteria in summer might be related to the generally higher biomass levels in summer. Bacteria which were significantly increased in summer included those involved in nitrogen or carbon cycles, such as Nitrospirae and Nitrospira, which are important nitrite-oxidizing bacteria. The phylum Spirochaetae is mainly composed of anaerobic heterotrophic microorganisms, which are involved in lignocellulose degradation and nitrogen fixation (Chen et al., 2017a). Chlorobi is a strictly anaerobic photoautotrophic bacterium and able to fix $\mathrm{CO}_{2}$. The higher input rates of $\mathrm{N}$ and $\mathrm{P}$, the lower the relative abundance of Chlorobi (Ling et al., 2017). The phylum Ignavibacteria consists of strictly organotrophic, facultatively anaerobic bacteria which are divided from Chlorobi. They play an important role in the degradation of polycyclic aromatic hydrocarbons (PAHs) and in the removal or organic matter (Kadnikov et al., 2013). Some bacteria in Hydrogenedent are involved in key geochemical processes including sulfur, nitrogen, and carbon cycles and generally survive in dark and hypoxic sub-surface biospheres (Momper et al., 2018). Most of the genera with significantly increased abundances in summer are thermophilic microorganisms. For example, the optimum growth temperature of Deferrisoma is $50^{\circ} \mathrm{C}$, while both Nitrospira and Draconibacterium can survive temperatures above $30^{\circ} \mathrm{C}$, corresponding to the relatively high temperatures in summer (Du et al., 2015; Hoshino et al., 2016; Daims and Wagner, 2018).

The bacterial species with increased abundances in the other seasons were mainly aerobic bacteria and involved in the degradation of cellulose and chitosan. In winter, the abundance of Actinobacteria, which can adapt to lower temperatures, was significantly increased, and they played important roles in carbon cycling and the decomposition of cellulose and chitosan (Aparicio et al., 2018). Armatimonadetes, a group of aerobic oligotrophic soil bacteria with a wide distribution, was chemoautotrophic and involved in the degradation of photosynthetic biomass (Hu et al., 2014). Previous studies have found that Armatimonadetes might also be involved in hydrolysis-fermentation and the degradation of cellulose (Lee et al., 2014). The abundance of genera Lutibacter and Aquibacter in the family Flavobacteriaceae are also significantly increased in the winter. Flavobacteriaceae can degrade high-molecular-weight organic polymers, including cellulose, chitosan, and proteins (Wissuwa et al., 2017), while Kangiella can 
use lignocellulose degrade by-products derived from it, facilitating the degradation of rhizome residues in sediments (Darjany et al., 2014). The increased abundances of these bacteria in winter might be related to the lower temperatures, decreased organic nutrient content, increased dissolved oxygen levels, as well as the relatively higher contents of animal and plant residues. Gemmatimonadetes, with a significantly higher abundance in autumn, are common soil bacteria and can adapt to low humidity levels; they are an indicator for semiarid ecosystems (Genderjahn et al., 2018); this corresponds to the decrease in relative humidity in autumn. Halioglobus, with a significantly increased abundance in spring samples, is a Gram-negative aerobic bacterium and can reduce nitrate into $\mathrm{N}_{2}$ (Chen et al., 2017b).

\section{Conclusion}

The microbial community structure in the Sansha Bay sediments presented significant seasonal variations, with the most significant differences in summer. The environmental factors driving this microbial community variation were mainly temperature, but also organic carbon and total nitrogen concentrations. However, no significant differences were observed in physicochemical indexes among different seasons, this indicated that the bacterial communities are more sensitive and can serve as a good sentinel for environmental changes. The results present here provide microbial parameters for assessing the environment quality, and to lay a foundation for further illumination of the ecological functions of microorganisms in intertidal sediments. However, most of our discussions regarding the bacterial functions derived from previous studies, clearly a combination of the high throughput sequencing approach presented here and isolation and characterization of the indicator bacteria will both be required in the future to better understand microbial functionality. Furthermore, numerous microbial groups detected in this study were uncategorized or unidentified, and their physiological functions needed to be further investigated.

Acknowledgments. The authors would like to thank the NSFC project (31272669) and the Public Science and Technology Research Funds Projects of Ocean (201205009-4) for funding support for the present research.

\section{REFERENCES}

[1] Aparicio, J. D., Saez, J. M., Raimondo, E. E., Benimeli, C. S., Polti, M. A. (2018): Comparative study of single and mixed cultures of actinobacteria for the bioremediation of co-contaminated matrices. - Journal of Environmental Chemical Engineering 6(2): S2213343718301519.

[2] Arsad, A., Abustan, I., Rawi, C. S., Syafalni, S. (2012): Integrating Biological Aspects into River Water Quality Research in Malaysia: An Opinion. - Social Science Electronic Publishing.

[3] Binladen, J., Gilbert, M. T. P., Bollback, J. P., Panitz, F., Bendixen, C., Nielsen, R., Willerslev, E. (2007): The use of coded PCR primers enables high-throughput sequencing of multiple homolog amplification products by 454 parallel sequencing. - Plos One 2(2): e197.

[4] Bradstreet, R. B. (1954): Kjeldahl Method for Organic Nitrogen. - Analytical Chemistry 26(1): 185-187. 
[5] Cabana, D., Sigala, K., Nicolaidou, A., Reizopoulou, S. (2013): Towards the implementation of the Water Framework Directive in Mediterranean transitional waters: the use of macroinvertebrates as biological quality elements. - Advances in Oceanography \& Limnology 4(2): 212-240.

[6] Cao, Y., Cherr, G. N., Córdova-Kreylos, A. L., Fan, T. W.-M., Green, P. G., Higashi, R. M., LaMontagne, M. G., Scow, K. M., Vines, C. A., Yuan, J., Holden, P. A. (2006): Relationships between Sediment Microbial Communities and Pollutants in Two California Salt Marshes. - Microbial Ecology 52(4): 619-633.

[7] Caporaso, J. G., Gordon, J. I. (2011): Global patterns of 16s rrna diversity at a depth of millions of sequences per sample. - Proc Natl Acad Sci USA 108.

[8] Chen, C., Xu, X. J., Xie, P., Yuan, Y., Zhou, X., Wang, A. J., Lee, D. J., Ren, N. Q. (2017a): Pyrosequencing reveals microbial community dynamics in integrated simultaneous desulfurization and denitrification process at different influent nitrate concentrations. - Chemosphere 171: 294-301.

[9] Chen, Z., Wuang, G., Dan, W., Fang-Kun, S. I. (2017b): Analysis on Change Characteristic of Autumn Relative Humidity in China. - Modern Agricultural Science \& Technology.

[10] Daby, D. (2006): Coastal Pollution and Potential Biomonitors of Metals in Mauritius. Water Air \& Soil Pollution 174(1-4): 63-91.

[11] Daims, H., Wagner, M. (2018): Nitrospira. - Trends in Microbiology 26(5): 462-463.

[12] Darjany, L. E., Whitcraft, C. R., Dillon, J. G. (2014): Lignocellulose-responsive bacteria in a southern California salt marsh identified by stable isotope probing. - Frontiers in Microbiology 5(6): 263.

[13] Du, J., Lai, Q., Xie, Y., Liu, Y., Shao, Z., Dong, C. (2015): Draconibacterium sediminis sp. nov., isolated from river sediment. - International Journal of Systematic \& Evolutionary Microbiology 65(7): 2310.

[14] Genderjahn, S., Alawi, M., Mangelsdorf, K., Horn, F., Wagner, D. (2018): Desiccationand Saline-Tolerant Bacteria and Archaea in Kalahari Pan Sediments. - Frontiers in Microbiology 9.

[15] Hannah, T. I. (2015): Influence of landscape- and stand-scale factors on avian communities in open pine ecosystems. - Dissertations \& Theses - Gradworks.

[16] Hicks, N., Liu, X., Gregory, R., Kenny, J., Lucaci, A., Lenzi, L., Paterson, D. M., Duncan, K. R. (2018): Temperature driven changes in benthic microbial diversity influences biogeochemical cycling in coastal sediments. - Frontiers in Microbiology 9.

[17] Hoshino, T., Kuratomi, T., Morono, Y., Hori, T., Oiwane, H., Kiyokawa, S., Inagaki, F. (2016): Ecophysiology of ZetaproteobacteriaAssociated with Shallow Hydrothermal IronOxyhydroxide Deposits in Nagahama Bay of Satsuma Iwo-Jima, Japan. - Frontiers in Microbiology 6(386): 1554.

[18] Hu, Z. Y., Wang, Y. Z., Im, W. T., Wang, S. Y., Zhao, G. P., Zheng, H. J., Quan, Z. X. (2014): The First Complete Genome Sequence of the Class Fimbriimonadia in the Phylum Armatimonadetes. - Plos One 9(6): e100794.

[19] Huang, X. F. (2000): Survey, Observation and Analysis of Lake Ecology. - China Standard Press, Beijing.

[20] Kadnikov, V. V., Mardanov, A. V., Podosokorskaya, O. A., Gavrilov, S. N., Kublanov, I. V., Beletsky, A. V., Bonch-Osmolovskaya, E. A., Ravin, N. V. (2013): Genomic analysis of Melioribacter roseus, facultatively anaerobic organotrophic bacterium representing a novel deep lineage within Bacteriodetes/Chlorobi group. - Plos One 8(1): e53047.

[21] Lage, O. M., Bondoso, J. (2011): Planctomycetes diversity associated with macroalgae. Fems Microbiology Ecology 78(2): 366-375.

[22] Lee, C. Y., Morgan, X. C., Dunfield, P. F., Tamas, I., Mcdonald, I. R., Stott, M. B. (2014): Genomic analysis of Chthonomonas calidirosea, the first sequenced isolate of the phylum Armatimonadetes. - Isme Journal 8(7): 1522-1533. 
[23] Legendre, P., Fortin, M., Borcard, D. (2016): Should the mantel test be used in partial analysis? - Methods Ecol. Evol. 6(11): 1239-1247.

[24] Ling, N., Chen, D., Guo, H., Wei, J., Bai, Y., Shen, Q., Hu, S. (2017): Differential responses of soil bacterial communities to long-term $\mathrm{N}$ and $\mathrm{P}$ inputs in a semi-arid steppe. - Geoderma 292: 25-33.

[25] Magurran, A. E. (1988): Ecological diversity and its measurement. - Princeton, NJ: Princeton University Press.

[26] Momper, L., Aronson, H. S., Amend, J. P. (2018): Genomic description of 'Candidatus Abyssubacteria' a novel subsurface lineage within the candidate phylum Hydrogenedentes. - Frontiers in Microbiology 9.

[27] Okeke, B. C., Thomson, M. S., Moss, E. M. (2011): Occurrence, molecular characterization and antibiogram of water quality indicator bacteria in river water serving a water treatment plant. - Science of The Total Environment 409(23): 4979-4985.

[28] R Development Core Team (2008): R: A language and environment for statistical computing. - In: R Foundation for Statistical Computing, Vienna, Austria, (ISBN 3900051-07-0).

[29] Shabarova, T., Kasalický, V., Šimek, K., Nedoma, J., Znachor, P., Posch, T., Pernthaler, J., Salcher, M. M. (2017): Distribution and ecological preferences of the freshwater lineage LimA (genus Limnohabitans) revealed by a new double hybridisation approach. Environmental Microbiology 19(3): 1296.

[30] Simpson, E. H. (1949): Measurement of diversity. - Nature 163: 688.

[31] Sun, M. Y., Dafforn, K. A., Brown, M. V., Johnston, E. L. (2012): Bacterial communities are sensitive indicators of contaminant stress. - Marine Pollution Bulletin 64(5): 10291038.

[32] Tang, F. Y., Wang, J. H., Chen, H., Hang, B. L., Chen, Y. (2018): Community structure of phytoplankton and its relationship with environmental factors of Sansha Bay in spring. - Journal Of Shanghai Ocean Uninversuty 04: 522-530. (in Chinese).

[33] Van den Wollenberg, A. L. (1977): Redundancy analysis an alternative for canonical correlation analysis. - Psychometrika 42(2): 207-219.

[34] Viswanathan, S., Voss, K. A., Pohlman, A., Gibson, D., Purohit, J. (2010): Evaluation of the biocriteria of streams in the San Diego Hydrologic Region. - Journal of Environmental Engineering 136(6): 627-637.

[35] Wang, L., Wang, C., Deng, D. G., Zhao, X. X., Zhou, Z. Z. (2015): Temporal and spatial variations in phytoplankton: correlations with environmental factors in Shengjin Lake, China. - Environmental Science \& Pollution Research 22(18): 1-13.

[36] Wang, N., Xiong, J. Q., Wang, X. C., Zhang, Y., Liu, H., Zhou, B., Pan, P., Liu, Y., Ding, F. (2018): Relationship between phytoplankton community and environmental factors in landscape water with high salinity in a coastal city of China. - Environmental Science and Pollution Research 25(28): 28460-28470.

[37] Wissuwa, J., Le Moine Bauer, S., Steen, I. H., Stokke, R. (2017): Complete genome sequence of Lutibacter profundi LP1 T isolated from an Arctic deep-sea hydrothermal vent system. - Standards in Genomic Sciences 12(1): 5.

[38] Zhao, L., Zhu, L., Liu, G. (2010): Relationships between Sedimentary Microbial Communities and Nutrient Factors in Luoma Lake. - International Conference on Bioinformatics \& Biomedical Engineering. 


\section{APPENDIX}

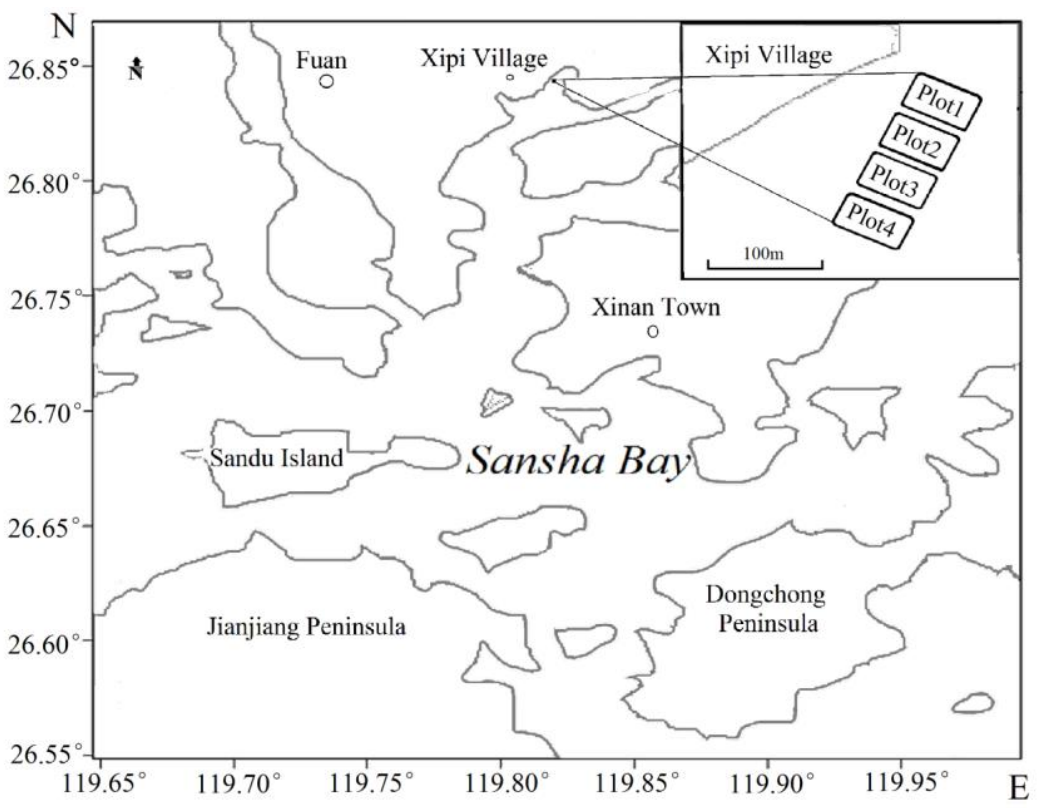

Figure A1. Location map of the sampling

Table A1. $P$ values of diversity indices of bacterial communities in mudflat sediments of Sansha Bay

\begin{tabular}{c|c|c|c|c|c|c}
\hline Estimators & $\begin{array}{c}\text { P value } \\
\text { (autumn-spring) }\end{array}$ & $\begin{array}{c}\text { P value } \\
\text { (autumn-winter) }\end{array}$ & $\begin{array}{c}\text { P value } \\
\text { (autumn-summer) }\end{array}$ & $\begin{array}{c}\text { P value } \\
\text { (spring-winter) }\end{array}$ & $\begin{array}{c}\text { P value } \\
\text { (spring-summer) }\end{array}$ & $\begin{array}{c}\text { P value } \\
\text { (summer-winter) }\end{array}$ \\
\hline OUT & 0.872 & 0.06539 & 0.4479 & 0.1103 & 0.5368 & 0.4972 \\
Shannon & 0.5452 & 0.3713 & 0.7071 & 0.2127 & 0.467 & 0.7874 \\
Simpson & 0.1555 & 0.9517 & 0.9806 & 0.2721 & 0.4066 & 0.9868 \\
ACE & 0.8695 & 0.1894 & 0.6641 & 0.191 & 0.7384 & 0.5481 \\
Chao1 & 0.6803 & 0.1583 & 0.5454 & 0.2064 & 0.7273 & 0.5859 \\
Coverage & 0.9778 & 0.07192 & 0.1153 & 0.3892 & 0.08455 & 0.8638 \\
PD & 0.8402 & 0.1099 & 0.659 & 0.1079 & 0.5739 & 0.4257 \\
\hline
\end{tabular}

OUT, Shannon, Simpson, ACE, Chao1, PD, Coverage represent operational taxonomic unit, the Shannon diversity index, the Simpson diversity index, the ACE estimator, the Chaol estimator, Phylogenetic diversity, and the Good's coverage, respectively. A $p$-value less than 0.05 was considered statistically significant differences

Table A2. $P$ values of significantly different microbial communities in mudflat sediments of Sansha Bay, phylum level

\begin{tabular}{cc|cc|cc}
\hline Species name & P value & Species name & P value & Species name & P value \\
\hline Nitrospirae & $1.26 \mathrm{E}-05$ & Hydrogenedentes & 0.001225 & Planctomycetes & 0.02849 \\
Lentisphaerae & 0.000177 & Tenericutes & 0.001299 & Chlorobi & 0.03041 \\
Acidobacteria & 0.000496 & Spirochaetae & 0.001386 & PAUC34f & 0.03488 \\
Gemmatimonadetes & 0.001011 & Ignavibacteriae & 0.01015 & Armatimonadetes & 0.04917 \\
Actinobacteria & 0.001032 & KSB3_Modulibacteria & 0.01223 & & \\
\hline
\end{tabular}


Table A3. $P$ values of significantly different microbial communities in mudflat sediments of Sansha Bay, genus level

\begin{tabular}{|c|c|c|c|c|c|}
\hline Species name & $P$ value & Species name & $P$ value & Species name & $P$ value \\
\hline Nitrospira & $1.26 \mathrm{E}-05$ & c_R76-B128 & 0.005521 & f__Flammeovirgaceae & 0.021896 \\
\hline Draconibacterium & 0.000204 & f__UASB-TL25 & 0.005604 & f__MidBa8 & 0.022372 \\
\hline f__P3OB-42 & 0.000224 & 3A7_sediment_group & 0.005799 & f__Flammeovirgaceae & 0.022533 \\
\hline f_JTB255_marine_benthic_group & 0.000369 & Pelagibius & 0.007134 & H16 & 0.022659 \\
\hline f__Nitrosomonadaceae & 0.000449 & Haliea & 0.007633 & Fusibacter & 0.02294 \\
\hline Gammaproteobacteria & 0.000968 & f__BSV26 & 0.007774 & c__WCHB1-41 & 0.03256 \\
\hline f__Cryomorphaceae & 0.001018 & f__MBAE14 & 0.009141 & Desulfatiglans & 0.03713 \\
\hline Deferrisoma & 0.001846 & o__ Sva0485 & 0.009547 & Lutibacter & 0.039 \\
\hline Spirochaeta & 0.001886 & $\mathrm{p} \_$B Bacteroidetes & 0.021101 & Nitrospina & 0.04354 \\
\hline f__Xanthomonadales_Incertae_Sedis & 0.002456 & Haliangium & 0.021237 & Actibacter & 0.049079 \\
\hline c_Alphaproteobacteria & 0.003124 & c__Bacteroidetes_BD2-2 & 0.0213 & SEEP-SRB1 & 0.049361 \\
\hline o__BD7-8_marine_group & 0.00314 & f__Lentimicrobiaceae & $0.021342 \mathrm{c}$ & c_Gammaproteobacteria & 0.049369 \\
\hline c__Gemmatimonadetes & 0.003308 & Kangiella & 0.021679 & f__Nitrospinaceae & 0.049734 \\
\hline f__Oceanospirillaceae & 0.003574 & Robiginitalea & 0.021688 & f__Hydrogenophilaceae & 0.049901 \\
\hline Sulfurovum & 0.003582 & o__MSBL9 & 0.021718 & & \\
\hline f__OM1_clade & 0.004208 & Aquibacter & 0.021727 & & \\
\hline o_E01-9C-26_marine_group & 0.005462 & Halioglobus & 0.021768 & & \\
\hline
\end{tabular}

Table A4. P values of physicochemical indices of mudflat sediments in Sansha Bay

\begin{tabular}{c|c|c|c|c|c|c}
\hline Estimators & $\begin{array}{c}\text { P value } \\
\text { (autumn-spring) }\end{array}$ & $\begin{array}{c}\text { P value } \\
\text { (autumn-winter) }\end{array}$ & $\begin{array}{c}\text { P value } \\
\text { (autumn-summer) }\end{array}$ & $\begin{array}{c}\text { P value } \\
\text { (spring-winter) }\end{array}$ & $\begin{array}{c}\text { P value } \\
\text { (spring-summer) }\end{array}$ & $\begin{array}{c}\text { P value } \\
\text { (summer-winter) }\end{array}$ \\
\hline $\mathrm{pH}$ & 0.0574 & 0.5801 & 0.0837 & 0.0571 & 0.1535 & 0.0840 \\
$\mathrm{OrgC}$ & 0.5801 & 0.1396 & 0.0500 & 0.1070 & 0.1007 & 0.0563 \\
$\mathrm{OrgS}$ & 0.4003 & 0.5565 & 0.1755 & 0.1318 & 0.5527 & 0.0534 \\
$\mathrm{TP}$ & 0.0503 & 0.0545 & 0.0572 & 0.5966 & 0.6832 & 0.5979 \\
$\mathrm{TN}$ & 0.5598 & 0.1488 & 0.0975 & 0.4626 & 0.0599 & 0.0513 \\
$\mathrm{Tm}$ & 0.0559 & 0.0594 & 0.0503 & 0.0519 & 0.0503 & 0.0212 \\
\hline
\end{tabular}

$\mathrm{TN}, \mathrm{TP}, \mathrm{OrgC}$ and $\mathrm{OrgS}, \mathrm{Tm}$ represent total nitrogen, total phosphorus, Organic carbon and sulfur contents, Water temperature, respectively

Table A5. $P$ values and envfit of Physicochemical indices in Sansha Bay

\begin{tabular}{c|c|c|c|c}
\hline Estimators & RDA1 & RDA2 & $\mathrm{r}^{2}$ & P value \\
\hline $\mathrm{pH}$ & -0.1782 & 0.984 & 0.5437 & 0.004 \\
OrgC & -0.9734 & 0.2292 & 0.6121 & 0.001 \\
OrgS & 0.7598 & -0.6501 & 0.2965 & 0.097 \\
$\mathrm{TP}$ & -0.5014 & 0.8652 & 0.4572 & 0.016 \\
$\mathrm{TN}$ & -0.9868 & -0.1622 & 0.6171 & 0.002 \\
$\mathrm{Tm}$ & -0.9042 & 0.4272 & 0.8758 & 0.001 \\
\hline
\end{tabular}

$\mathrm{TN}, \mathrm{TP}, \mathrm{OrgC}$ and $\mathrm{OrgS}, \mathrm{Tm}$ represent total nitrogen, total phosphorus, Organic carbon and sulfur contents, Water temperature, respectively 